Www.jmscr.igmpublication.org

Impact Factor (SJIF): 6.379

Index Copernicus Value: 79.54

ISSN (e)-2347-176x ISSN (p) 2455-0450

crossrefDOI: https://dx.doi.org/10.18535/jmscr/v6i10.107

Journal Of Medical Science And Clinical Research

\title{
Prevalence of depression among adult patients with type 2 diabetes in outpatient clinic at family medicine department SFH
}

\author{
Author \\ Layla Mansour Alotaibi* \\ *Family Medicine Resident
}

\begin{abstract}
:
Background: Both of Type 2 diabetes mellitus and depression are major public health problems. Diabetes mellitus is characterized by increased level of blood glucose, its prevalence is increasing globally. Depression is a psychological disorder that affects large number of population worldwide. Association between depression and type 2 diabetes mellitus was reported.

Aim: To define the prevalence of depression and its associated risk factors among adult patients with type 2 diabetes.

Method: This study is cross sectional single centre study, it was conducted on patients with type 2 diabetes at primary care center, Security Forces Hospital, Riyadh Saudi Arabia. The study was performed between the period from 1st of May 2017 to 1st of Jun 2017, using validated questionnaire. Statistical analysis and possible correlations were performed.

Results: Depression was prevalent in 55\% of Type2 Diabetes Mellitus patients, with more prevalence of simple depression (26.2\%). Different factors were associated with the prevalence of depression including personal characteristics such as age $(P$-value=0.005), gender $(P$-value $=0.005)$, educational level $(P$ value $=0.006)$ and monthly income $(P$-value $=0.0001)$ as well as clinical characteristics such as time of diagnosis $(P$-value $=0.023)$ and presence of chronic diseases $(P$-value $=0.027)$.

Conclusion: Depression was moderately prevalent among type 2 diabetic patients with more prevalence of simple depression. Age, gender, educational level, salary, time of disease diagnosis, and presence of chronic diseases were associated with depression prevalence.

Keywords: Depression prevalence, Type 2 diabetes, depression, Saudi Arabia.
\end{abstract}

\section{Introduction}

Diabetes mellitus (DM) is a disease in which the blood glucose level increases, it is associated with reduced quality of life ${ }^{[1]}$. The prevalence of DM is increasing globally, it was reported that $6.6 \%$ of individuals all over the world have DM and it will be rise in 2030 to $7.8 \%{ }^{[2]}$. It was reported that DM was prevalent among $25.4 \%$ of Saudi Population $^{[3]}$. Type 2 diabetes mellitus (T2DM) represents a major chronic health problem that afflicts the global population ${ }^{[4]}$, it accounts for 85$95 \%$ among all diabetes cases ${ }^{[5]}$. Both of T2DM and depression are major public health problems ${ }^{[6]}$. Depression is a serious and common mood disorder that represents with disturbed sleep or appetite, feeling guilt and loss of pleasure ${ }^{[7]}$, it affects 350 million individuals globally, it is responsible for moderate to severe disability ${ }^{[8]}$. In 
Saudi Arabia, depression affects $40 \%$ of general population ${ }^{[9]}$. Several studies showed that there was an association between depression and diabetes ${ }^{[10-12]}$. It was recognized that depression had an impact on the quality of life of diabetic patients $^{[13]}$. Another controversial studies were published $^{[14,15]}$. Depression is an independent risk factor for T2DM development ${ }^{[16]}$ and it was found that risk of DM increased by $65 \%$ in case of presence of significant depression ${ }^{[17]}$. It was reported that depression can be independent modifiable risk factor for progression of complication among T2DM patients ${ }^{[18]}$. Several studies reported a bidirectional relationship between diabetes and depression ${ }^{[19-21]}$. Depression is psychological disorder that can be evaluated by patient health questionnaire (PHQ) which is one of the most commonly used clinical diagnostic tool in primary care, it has shown reliability and efficiency for diagnosing depression and other disorders such as somatic disorders and anxiety $^{[22]}$. The validity of the Arabic version of PHQ in screening depression, anxiety, panic and somatic disorders was proven by Saudi study which was conducted in King Saud university recently in $2017^{[22]}$.

\section{Literature Review}

Diabetes mellitus is a risk factor for depression development, and depression acts as a risk factor for poor metabolic control in diabetic patients. It was stated that some investigators reported moderate to strong correlations between glycemic control and depression ${ }^{[23]}$. Identification of depression risk factors may help physicians to identify diabetic patients at risk of depression development earlier and hence improve its prevention and as a result decreasing the longterm complications ${ }^{[24]}$. It was reported in metaanalysis by Anderson et al that $30 \%$ of diabetic patients have depressive symptoms ${ }^{[10]}$, and more than $10 \%$ had major depression ${ }^{[10,25,26]}$.

A study from Jeddah ${ }^{[27]}$ assessed the prevalence of depression among diabetic and non-diabetic patients as well as the predictors of depression, it was found that depression and diabetes were associated with morbidity and early mortality. Another Saudi study reported that $50 \%$ of type 2 diabetic patients suffered depression ${ }^{[28]}$.

Furthermore a study from Al Qassim, Saudi Arabia showed lower prevalence of depression among T2DM patients, where $34.8 \%$ of patients were found to have depression ${ }^{[29]}$. Also, the authors of $\mathrm{Al}$ Qassim study found that depression was associated with social support and years living with diabetes ${ }^{[29]}$. Another study showed that in patients with T2DM, depression was found to be associated with increased risk of microvascular and macrovascular complications ${ }^{[30,31]}$ as well as poor glycemic control ${ }^{[32]}$.

A study done in Canada reported an incidence of depression among $4.3 \%$ of diabetic patients ${ }^{[24]}$. A study from India reported higher prevalence of $27.05 \%$ of depression among diabetic patients ${ }^{[23]}$. Another Indian study showed that depression was prevalent in $43.3 \%$ of diabetic patients ${ }^{[33]}$. In Sudanese study, there were $44 \%$ of type 2 diabetic patients had depression ${ }^{[1]}$. The aim of this study is to assess the prevalence of depression among type 2 diabetes mellitus patients and its associated risk factors.

\section{Patients \& Methods}

\section{Subjects and study design}

This is a cross sectional single center study which was conducted at PCC, Security Forces Hospital, Riyadh Saudi Arabia, in the period from 1st may 2017 to 1 st June 2017, the inclusion criteria were adult patients whose age was 18 years and more, the patients had type 2 diabetes mellitus. The exclusion criteria included patients who were using anti-depressant medications, having history of psychiatric illness other than depression and those unwilling to be part of the study were excluded. The study was performed using a validated questionnaire PHQ-9 which was translated into Arabic.

\section{Sample Size and Technique}

Using expected prevalence of knowledge was reported $49 \%$ as given in literature for calculating 
our sample size. Under the simple random sampling with margin of error at $5 \%$ and the confidence level at $95 \%$, we needed a sample of size 348. Allowing for $15 \%$ non-response rate the final required sample size is 200 . Our sample size was be restricted to the number of diabetic patients who attended family medicine clinic during 1 month period. We used the following formula

$n=z 2(1-p) d 2$

Where $\mathrm{n}=$ sample size, $\mathrm{z}=\mathrm{z}$ statistic for the level of confidence, $\mathrm{P}=$ expected prevalence and $\mathrm{d}=$ allowable error. This formula assumes that "P" and "d" are decimal values.

\section{Statistical Analysis}

SPSS version 25 statistical package was used for statistical analysis. For comparisons between the groups, $t$-test was used for continuous variables and $\times 2$ test was used for categorical variables. The relationship between social varibles, and other clinical characteristics (Medical history and Chronic diseases) was assessed by Pearson's correlation analysis and chi square test. Significance was determined at $P<0.05$.

\section{Results}

In the current study, depression was more prevalent among patients, there were 55\% suffered depression, figure1.

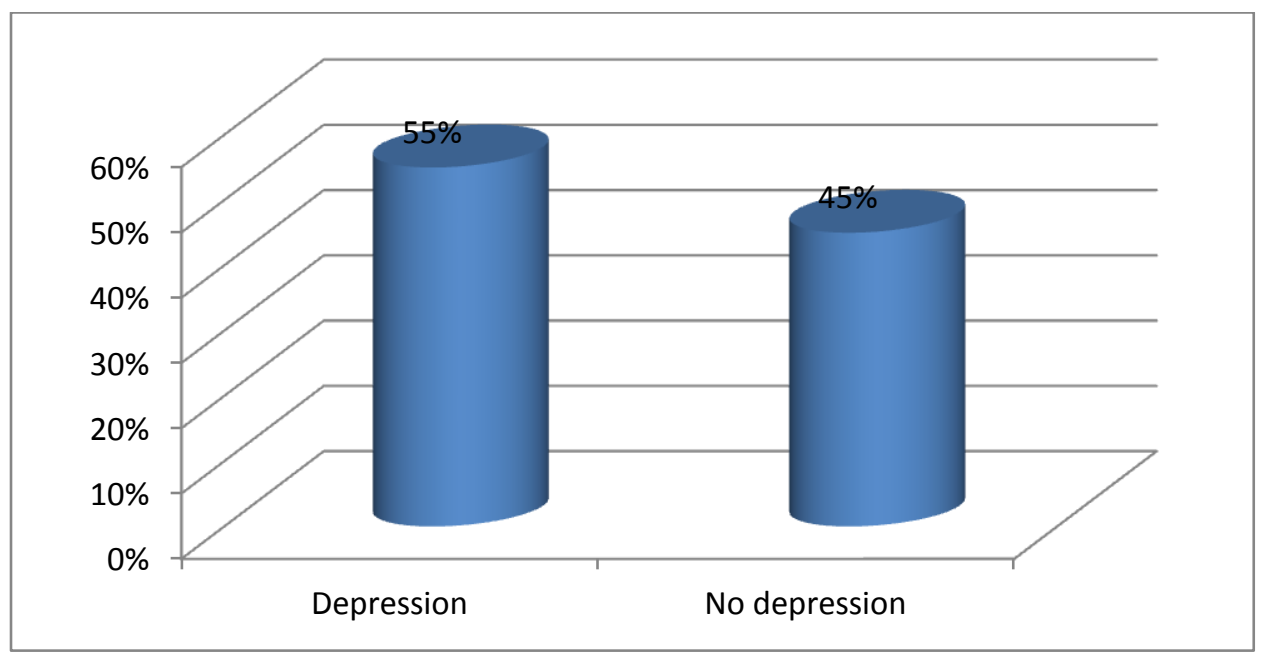

Fig 1: Prevalence of depression among participants

According to depression severity, there were $26.2 \%$ had mild depression, $16.9 \%$ had moderate depression, $7 \%$ and $1.9 \%$ had moderate to severe and severe depression respectively, figure 2 .

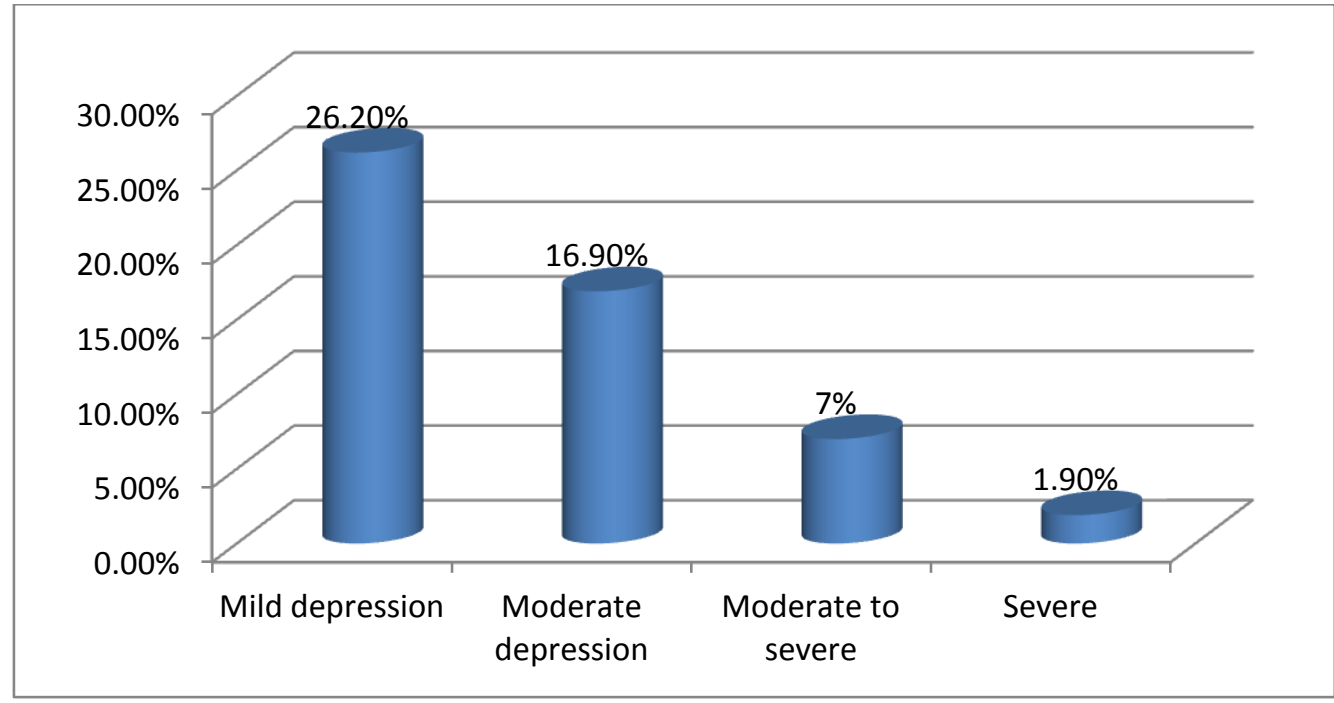

Fig 2: Prevalence of depression severity 
The correlations between depression prevalence and different variables were investigated, table 1 summarizes such correlations. Depression was significantly $(\mathrm{P}$-value $=0.005)$ more common among those older than 45 years and females (Pvalue $=0.005$ ). Marital status, employment status and type of treatment didn't influence prevalence of depression (P-value>0.05). Students of secondary school were more prone to suffer depression $(\mathrm{P}$-value $=0.006)$, as well as those with monthly income less than 8000SR (Pvalue $=0.000)$. Depression was significantly associated with time of diagnosis $(\mathrm{P}-\mathrm{value}=0.023$ ) those diagnosed since 5-10 years were more prone to suffer depression. Depression was also significantly associated with the presence of chronic diseases (P-value $=0.027$ ), depression was more common in those with hypothyroidism $(87.5 \%)$, followed by those with high blood lipid level $(66.7 \%)$ and rheumatism $(66.3 \%)$.

Table 1: Correlations between Prevalence of depression and different variables

\begin{tabular}{|c|c|c|c|}
\hline Variables & $\begin{array}{c}\text { No depression } \\
45 \%\end{array}$ & $\begin{array}{l}\text { Depression } \\
55 \%\end{array}$ & P-value \\
\hline $\begin{array}{l}\text { Age (years) } \\
<25 \text { years } \\
25-45 \text { years } \\
>45 \text { years }\end{array}$ & $\begin{array}{l}66.7 \% \\
51.4 \% \\
42.6 \%\end{array}$ & $\begin{array}{l}33.3 \% \\
48.6 \% \\
57.4 \%\end{array}$ & $0.005^{*}$ \\
\hline $\begin{array}{l}\text { Gender } \\
\text { Male } \\
\text { Female }\end{array}$ & $\begin{array}{l}52.1 \% \\
31.8 \%\end{array}$ & $\begin{array}{l}47.9 \% \\
68.2 \%\end{array}$ & $0.005^{*}$ \\
\hline $\begin{array}{l}\text { Marital status } \\
\text { Single } \\
\text { Married } \\
\text { Widowed } \\
\text { Divorce }\end{array}$ & $\begin{array}{c}42.9 \% \\
48.3 \% \\
20 \% \\
33.3 \%\end{array}$ & $\begin{array}{c}57.1 \% \\
51.7 \% \\
80 \% \\
66.7 \%\end{array}$ & 0.06 \\
\hline $\begin{array}{l}\text { Education level } \\
\text { Elementary } \\
\text { Intermediate } \\
\text { Secondary } \\
\text { Diploma } \\
\text { University } \\
\text { Graduated } \\
\end{array}$ & $\begin{array}{c}29.8 \% \\
51.4 \% \\
46.8 \% \\
50 \% \\
53.3 \% \\
85.7 \%\end{array}$ & $\begin{array}{c}70.2 \% \\
48.6 \% \\
53.2 \% \\
50 \% \\
46.7 \% \\
14.3 \% \\
\end{array}$ & $0.006^{*}$ \\
\hline $\begin{array}{l}\text { Employment status } \\
\text { Students } \\
\text { Employed } \\
\text { Unemployed } \\
\text { Pensions }\end{array}$ & $\begin{array}{l}66.7 \% \\
52.5 \% \\
28.2 \% \\
54.2 \%\end{array}$ & $\begin{array}{l}33.3 \% \\
47.5 \% \\
71.8 \% \\
45.8 \%\end{array}$ & 0.7 \\
\hline $\begin{array}{l}\text { Income/month } \\
<8000 \text { SR } \\
8000-11000 \text { SR } \\
12000-15000 \text { SR } \\
15000-20000 \text { SR } \\
>20000 \text { SR } \\
\end{array}$ & $\begin{array}{l}26.3 \% \\
57.1 \% \\
57.7 \% \\
52.6 \% \\
68.8 \% \\
\end{array}$ & $\begin{array}{l}73.7 \% \\
42.9 \% \\
42.3 \% \\
47.4 \% \\
31.2 \% \\
\end{array}$ & $0.000 *$ \\
\hline $\begin{array}{l}\text { Time of diagnosis } \\
<5 \text { years } \\
5-10 \text { years } \\
>15 \text { years }\end{array}$ & $\begin{array}{c}55.8 \% \\
39.7 \% \\
41 \%\end{array}$ & $\begin{array}{c}44.2 \% \\
60.3 \% \\
59 \%\end{array}$ & $0.023 *$ \\
\hline $\begin{array}{l}\text { Type of treatment } \\
\text { Tablet } \\
\text { Insulin injection } \\
\text { Both }\end{array}$ & $\begin{array}{c}50 \% \\
37.2 \% \\
43.4 \%\end{array}$ & $\begin{array}{c}50 \% \\
62.8 \% \\
56.6 \%\end{array}$ & 0.2 \\
\hline $\begin{array}{l}\text { Chronic diseases } \\
\text { Hypertension } \\
\text { High blood lipid level } \\
\text { Heart disease } \\
\text { Hypothyroidism } \\
\text { Rheumatism } \\
\text { Other chronic diseases }\end{array}$ & $\begin{array}{c}38.7 \% \\
33.3 \% \\
75 \% \\
12.5 \% \\
33.3 \% \\
57.1 \% \\
\end{array}$ & $\begin{array}{l}61.3 \% \\
66.7 \% \\
25 \% \\
87.5 \% \\
66.3 \% \\
42.9 \% \\
\end{array}$ & $0.027 *$ \\
\hline
\end{tabular}


Table 1: Depression by demographics and social variables

\begin{tabular}{|c|c|c|c|c|c|c|c|c|}
\hline \multirow[t]{2}{*}{ Variables } & \multicolumn{2}{|c|}{ No Depression } & \multicolumn{2}{|c|}{ Depression } & & & & \\
\hline & No & $\%$ & No & $\%$ & & & & \\
\hline No (209) missing(4) & 95 & 45 & 114 & 55 & & & & \\
\hline \multicolumn{9}{|l|}{ Age in years } \\
\hline Less than 25 years & 2 & 66.7 & 1 & 33.3 & \multirow{2}{*}{$\chi^{2}$} & Value: & \multicolumn{2}{|c|}{1.546} \\
\hline 25 to 45 years & 19 & 51.4 & 18 & 48.6 & & P-value: & \multicolumn{2}{|c|}{.462} \\
\hline More than 45 & 72 & 42.6 & 97 & 57.4 & \multicolumn{2}{|c|}{$\begin{array}{c}\text { Spearman correlation: } \\
.205 \\
\end{array}$} & \multicolumn{2}{|c|}{ P-value: .005 } \\
\hline \multicolumn{9}{|l|}{ Gender } \\
\hline Male & 49 & 52.1 & 45 & 47.9 & \multirow{2}{*}{$\chi^{2}$} & Value: & \multicolumn{2}{|c|}{7.681} \\
\hline Female & 28 & 31.8 & 60 & 68.2 & & P-value: & \multicolumn{2}{|c|}{.006} \\
\hline & & & & & \multicolumn{2}{|c|}{$\begin{array}{c}\text { Spearman correlation: } \\
0.205\end{array}$} & \multicolumn{2}{|c|}{ P-value:0.005 } \\
\hline \multicolumn{9}{|l|}{ Marital status } \\
\hline Single & 3 & 42.9 & 4 & 57.1 & \multirow{2}{*}{$\chi^{2}$} & Value: & \multicolumn{2}{|c|}{7.280} \\
\hline Married & 86 & 48.3 & 92 & 51.7 & & P-value: & \multicolumn{2}{|c|}{0.063} \\
\hline Widowed & 5 & 20 & 20 & 80 & Spea & orrelation: & & \\
\hline Divorce & 1 & 33.3 & 2 & 66.7 & & & P-valu & $: 0.003$ \\
\hline Educational level & & & & & & & & \\
\hline Elementary & 17 & 29.8 & 40 & 70.2 & & Value: & 11. & 509 \\
\hline Intermediate & 19 & 51.4 & 18 & 48.6 & $\chi^{-}$ & P-value: & 0.0 & \\
\hline Secondary & 29 & 46.8 & 33 & 53.2 & Spea & orrelation: & D & \\
\hline Diploma & 6 & 50 & & 50 & & & P-valu & $: 0.000$ \\
\hline University & 16 & 53.3 & 14 & 46.7 & & & & \\
\hline Graduate Studies & 6 & 85.7 & 1 & 14.3 & & & & \\
\hline Employment status & & & & & & & & \\
\hline Student & 2 & 66.7 & 1 & 33.3 & & Value: & 12.514 & \\
\hline Employed & 32 & 52.5 & 29 & 47.5 & $\chi^{-}$ & P-value: & 0.005 & \\
\hline Unemployed & 20 & 28.2 & 51 & 71.8 & Spea & orrelation: & $P_{-}$all & ( 780 \\
\hline Pensions & 39 & 54.2 & 33 & 45.8 & & & P-valu & $: 0.189$ \\
\hline Income/month & & & & & & & & \\
\hline Less than $8000 \mathrm{SR}$ & 20 & 26.3 & 56 & 73.7 & $\gamma^{2}$ & Value: & 19. & 410 \\
\hline 8000-11000 SR & 28 & 57.1 & 21 & 42.9 & $\chi$ & P-value: & 0.0 & \\
\hline $12000-15000 \mathrm{SR}$ & 15 & 57.7 & 11 & 42.3 & Spea & prrelation: & & \\
\hline $15000-20000 \mathrm{SR}$ & 10 & 52.6 & 9 & 47.4 & & & P-valu & $: 0.000$ \\
\hline More than 20000 SR & 11 & 68.8 & 5 & 31.2 & & & & \\
\hline Medical history & & & & & & & & \\
\hline When was your diagnc & & & & & & & & \\
\hline & & & & & & Value: & & \\
\hline Less Than 5 years & 29 & 55.8 & 23 & 44.2 & $\chi$ & P-value: & 0.0 & \\
\hline $5-10$ years & 29 & 39.7 & 44 & 0.3 & Spea & orrelation: & & \\
\hline More than 15 years & 34 & 41 & 49 & 59 & & & P-valu & $: 0.023$ \\
\hline Type of treatment & & & & & & & & \\
\hline Tablet & 54 & 50 & 54 & 50 & & & & \\
\hline Insulin Injection & 16 & 37.2 & 27 & 62.8 & $\chi^{2}$ & Value: & & \\
\hline All the above & 23 & 43.4 & 30 & 56.6 & & P-value: & & \\
\hline & & & & & Spea & 6 & P-valu & 0.277 \\
\hline Chronic diseases & & & & & & & & \\
\hline Hypertension & 36 & 38.7 & 57 & 61.3 & & Value: & & \\
\hline High blood lipid level & 15 & 33.3 & 30 & 66.7 & $\chi^{2}$ & P-value: & & \\
\hline Heart disease & 3 & 75 & 1 & 25 & Spea & orrelation: & $\mathrm{P}_{-\mathrm{y}}$ & \\
\hline Hypothyroidism & 1 & 12.5 & 7 & 87.5 & & & P-valu & $: 0.021$ \\
\hline Rheumatism & 1 & 33.3 & 2 & 66.3 & & & & \\
\hline Other chronic diseases & 8 & 57.1 & 6 & 42.9 & & & & \\
\hline
\end{tabular}

$* \mathrm{P}<.05$ 
In Table 2, the proportion of people who do not suffer from depression is $44.6 \%$. Those who have a simple depression are $29.6 \%$ and those who suffer from average depression are $16.9 \%$ and the the proportion of people have Depression is moderate to severe is equal to 7 percent and the Severe depression prpoprtion is 1.9 percent Finally, $55.4 \%$ of the study sample suffers from depression at different levels
Table 2 The severity of depression

\begin{tabular}{|l|c|c|}
\hline & Frequency & Percent \\
\hline There is no depression & 95 & 44.6 \\
\hline Simple depression & 63 & 29.6 \\
\hline Depression is average & 36 & 16.9 \\
\hline $\begin{array}{l}\text { Depression is moderate } \\
\text { to severe }\end{array}$ & 15 & 7.0 \\
\hline Severe depression & 4 & 1.9 \\
\hline Total & 213 & 100.0 \\
\hline
\end{tabular}

\begin{tabular}{|c|c|c|}
\hline \multicolumn{2}{|l|}{ Notes } & \multirow[b]{2}{*}{ 11-JUL-2018 15:47:39 } \\
\hline Output Created & & \\
\hline \multicolumn{3}{|l|}{ Comments } \\
\hline \multirow[t]{6}{*}{ Input } & Data & C:IUsersldell|Desktop|الاكتثابو السكري.sav \\
\hline & Active Dataset & DataSet1 \\
\hline & Filter & <none> \\
\hline & Weight & $<$ none $>$ \\
\hline & Split File & $<$ none $>$ \\
\hline & $\begin{array}{l}\mathrm{N} \text { of Rows in Working Data } \\
\text { File }\end{array}$ & 213 \\
\hline \multirow[t]{2}{*}{ Missing Value Handling } & Definition of Missing & $\begin{array}{l}\text { User-defined missing values are treated } \\
\text { as missing. }\end{array}$ \\
\hline & Cases Used & $\begin{array}{l}\text { Statistics are based on all cases with } \\
\text { valid data. }\end{array}$ \\
\hline Syntax & & $\begin{array}{l}\text { FREQUENCIES VARIABLES=Age Sex } \\
\text { MaritalStatus Education Job Income } \\
\text { Data Type ChronicDiseases } \\
\text { /FORMAT=NOTABLE } \\
\text { /ORDER=ANALYSIS. }\end{array}$ \\
\hline \multirow[t]{2}{*}{ Resources } & Processor Time & 00:00:00.00 \\
\hline & Elapsed Time & 00:00:00.07 \\
\hline
\end{tabular}

\begin{tabular}{|c|c|c|}
\hline \multicolumn{2}{|l|}{ Notes } & \multirow[b]{2}{*}{ 11-JUL-2018 15:47:59 } \\
\hline Output Created & & \\
\hline \multicolumn{3}{|l|}{ Comments } \\
\hline \multirow[t]{6}{*}{ Input } & Data & C:IUsersldell|Desktop|الاكتثابو السكري.sav \\
\hline & Active Dataset & DataSet1 \\
\hline & Filter & $\langle$ none $>$ \\
\hline & Weight & <none> \\
\hline & Split File & <none $>$ \\
\hline & $\begin{array}{l}\mathrm{N} \text { of Rows in Working Data } \\
\text { File }\end{array}$ & 213 \\
\hline \multirow[t]{2}{*}{ Missing Value Handling } & Definition of Missing & $\begin{array}{l}\text { User-defined missing values are treated } \\
\text { as missing. }\end{array}$ \\
\hline & Cases Used & $\begin{array}{l}\text { Statistics are based on all cases with } \\
\text { valid data. }\end{array}$ \\
\hline Syntax & & $\begin{array}{l}\text { FREQUENCIES VARIABLES=Age Sex } \\
\text { MaritalStatus Education Job Income } \\
\text { Data Type ChronicDiseases } \\
\text { /FORMAT=NOTABLE } \\
\text { /BARCHART FREQ } \\
\text { /ORDER=ANALYSIS. }\end{array}$ \\
\hline \multirow[t]{2}{*}{ Resources } & Processor Time & 00:00:04.54 \\
\hline & Elapsed Time & 00:00:02.78 \\
\hline
\end{tabular}



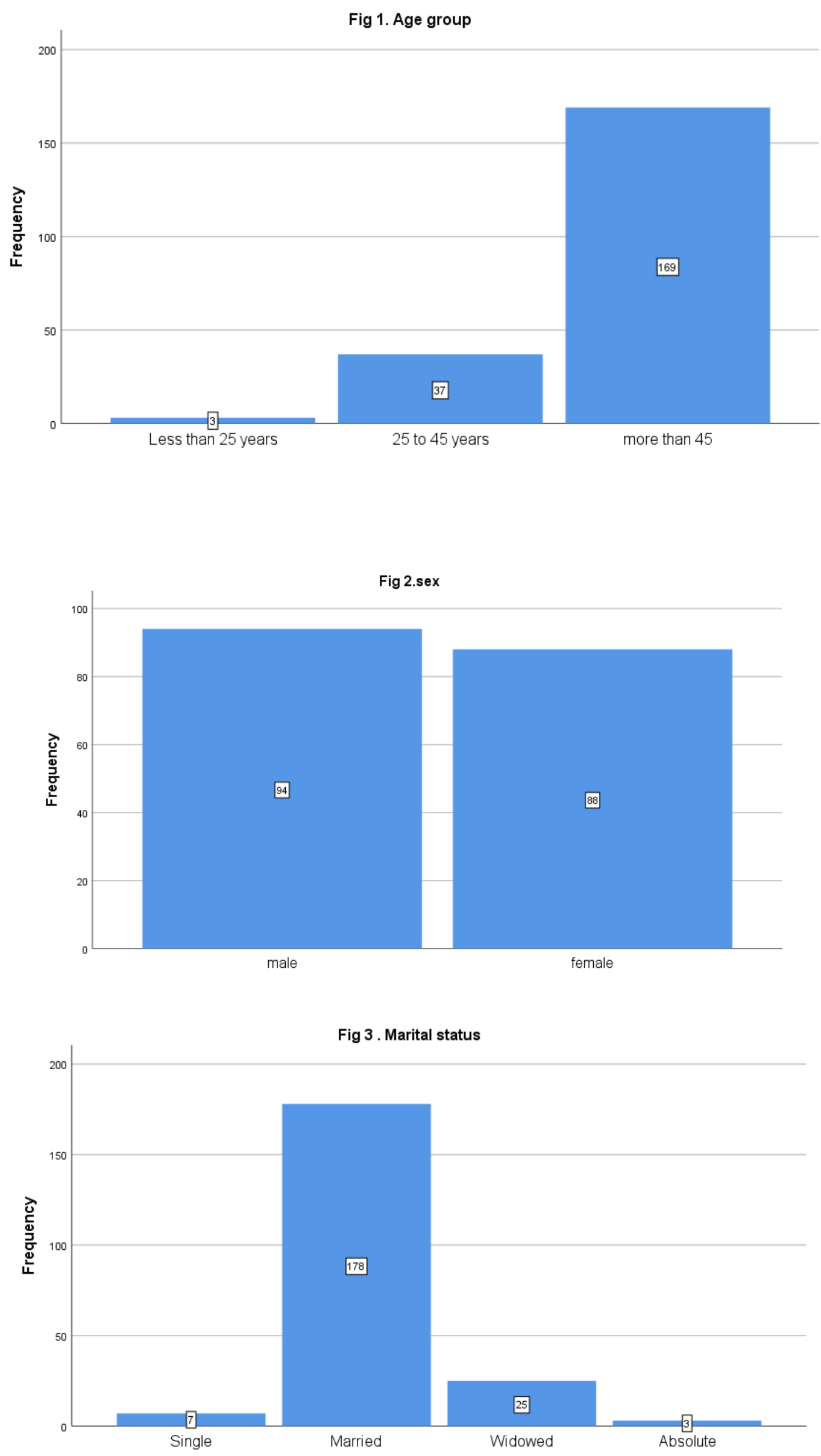

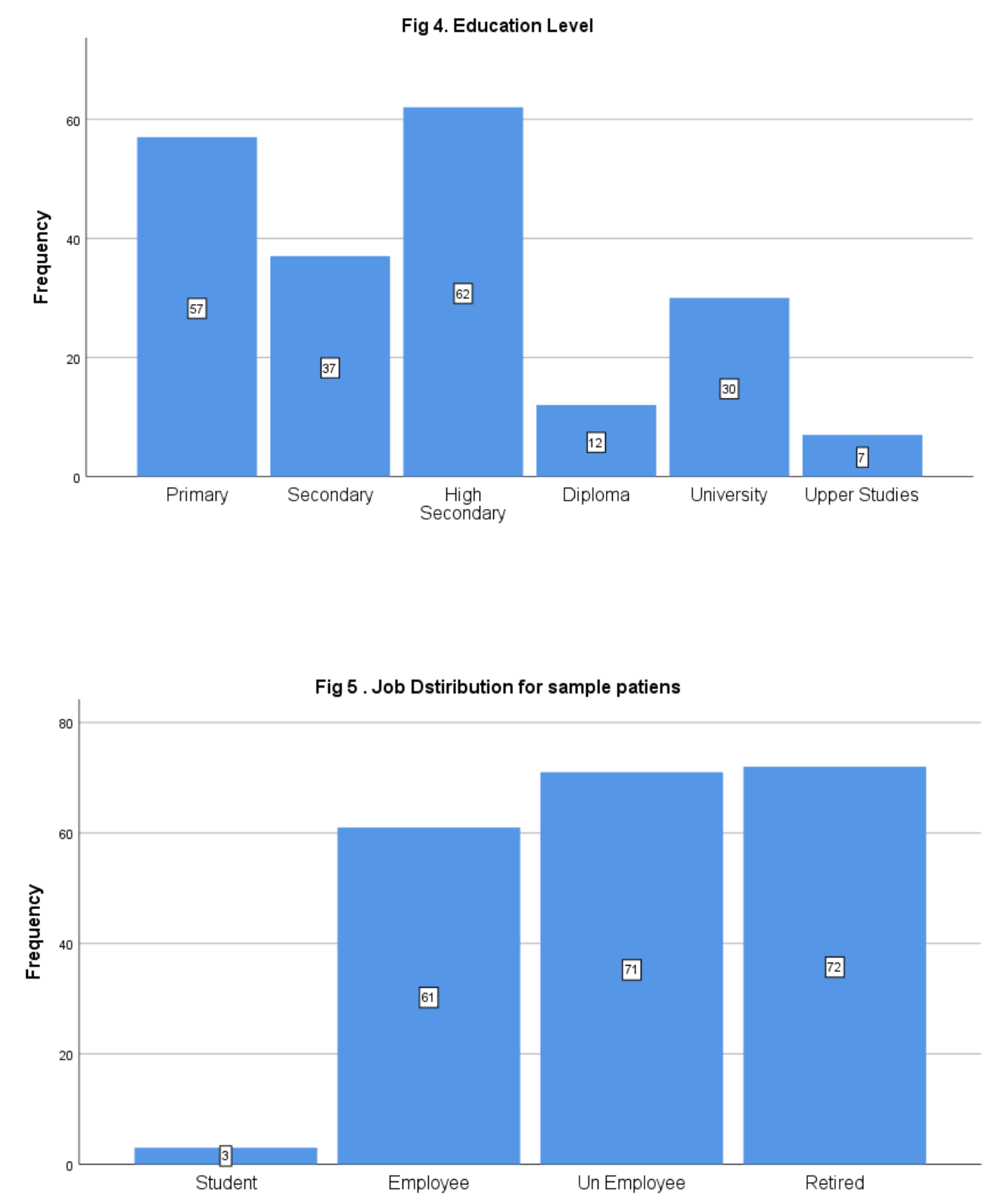

Fig 6. show the Income Level for pateint

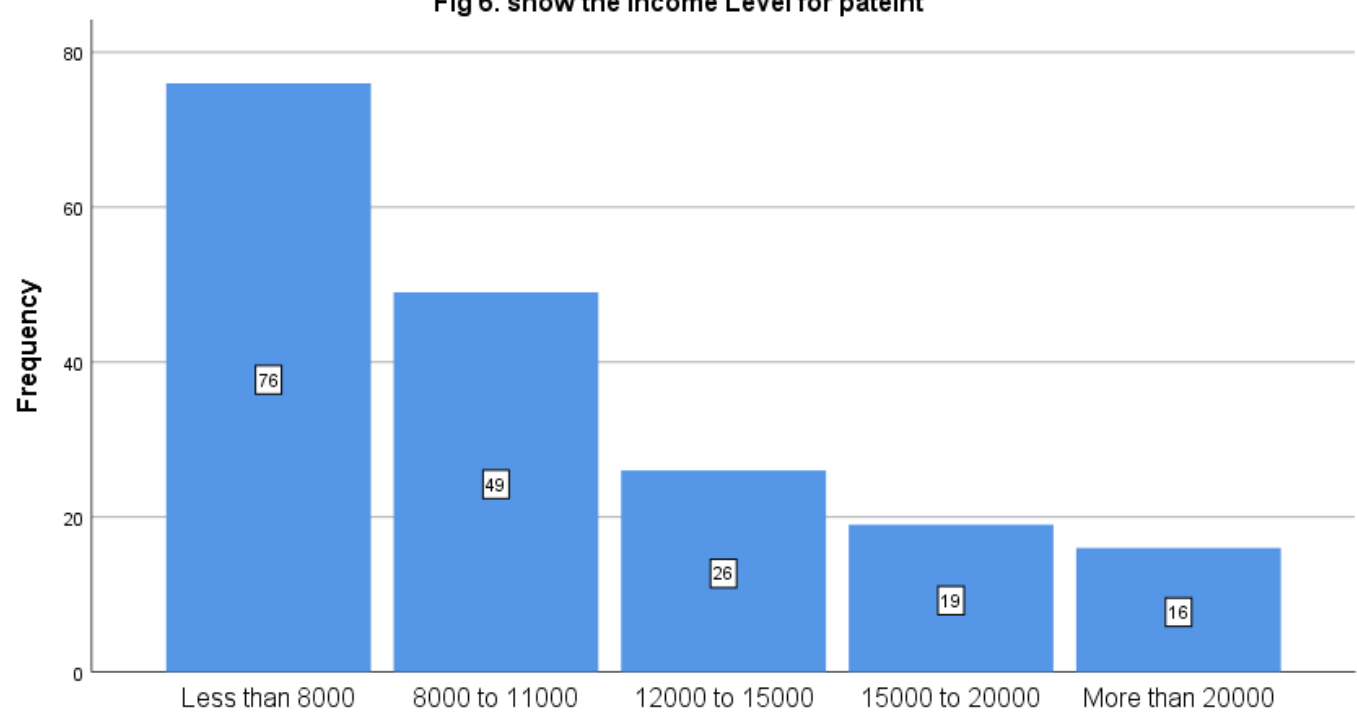



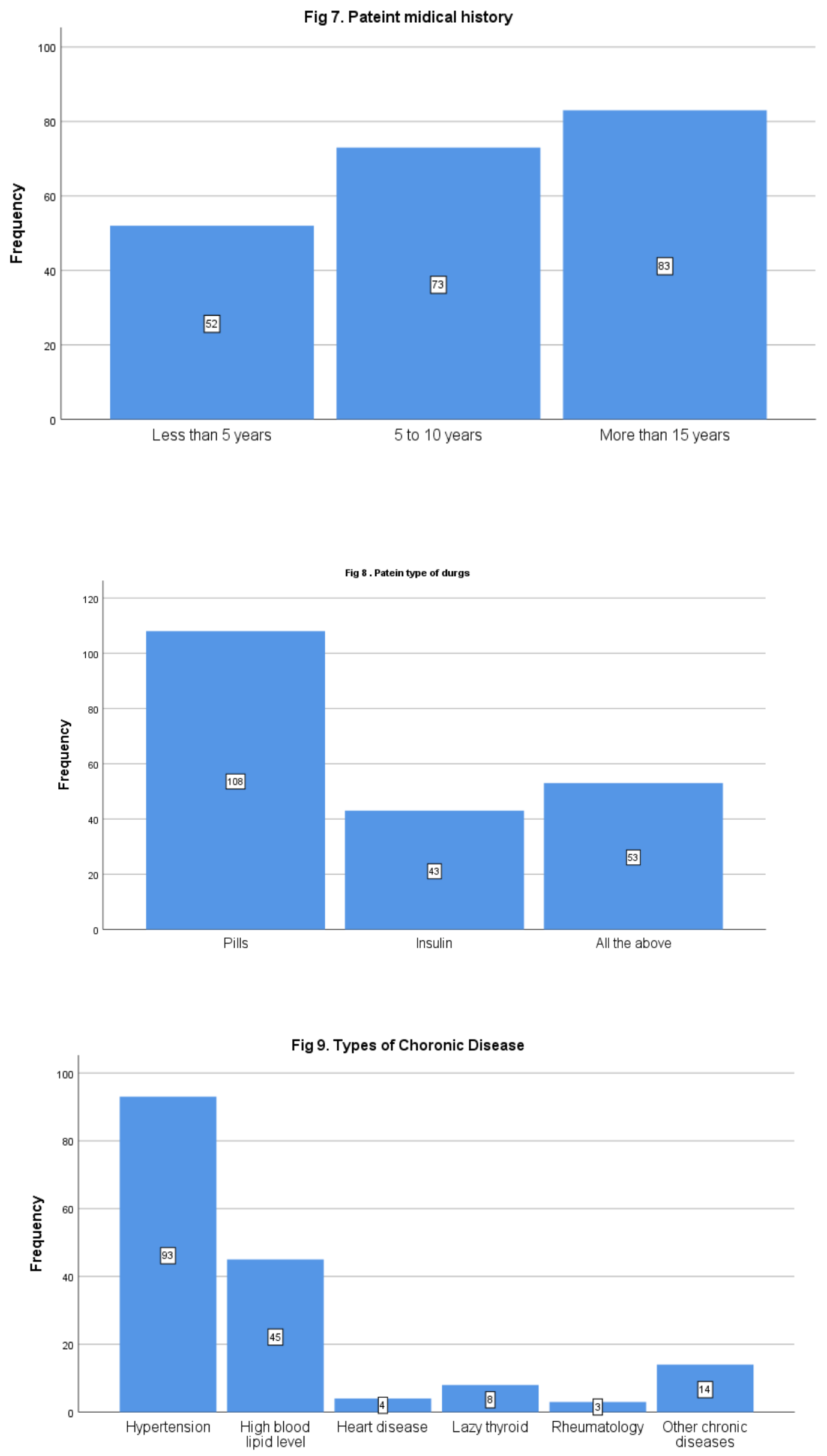


\begin{tabular}{|c|c|c|}
\hline \multicolumn{3}{|c|}{ Notes } \\
\hline \multicolumn{2}{|c|}{ Output Created } & 11-JUL-2018 15:49:07 \\
\hline \multicolumn{3}{|c|}{ Comments } \\
\hline \multirow[t]{6}{*}{ Input } & Data & $\begin{array}{l}\text { C:IUsersldell|Desktopl } \\
\text { الاكتئابو السكري.sav }\end{array}$ \\
\hline & Active Dataset & DataSet1 \\
\hline & Filter & $\langle$ none $>$ \\
\hline & Weight & $<$ none $>$ \\
\hline & Split File & $\langle$ none $>$ \\
\hline & $\begin{array}{c}\mathrm{N} \text { of Rows in Working Data } \\
\text { File }\end{array}$ & 213 \\
\hline \multirow[t]{2}{*}{ Missing Value Handling } & Definition of Missing & $\begin{array}{l}\text { User-defined missing values } \\
\text { are treated as missing. }\end{array}$ \\
\hline & Cases Used & $\begin{array}{l}\text { Statistics are based on all cases } \\
\text { with valid data. }\end{array}$ \\
\hline \multicolumn{2}{|c|}{ Syntax } & $\begin{array}{c}\text { FREQUENCIES } \\
\text { VARIABLES=a1 a2 a3 a4 a5 } \\
\text { a6 a7 a8 a9 Total b1 depression } \\
\text { /FORMAT=NOTABLE } \\
\text { /BARCHART FREQ } \\
\text { /ORDER=ANALYSIS. }\end{array}$ \\
\hline \multirow[t]{2}{*}{ Resources } & Processor Time & 00:00:03.96 \\
\hline & Elapsed Time & 00:00:02.02 \\
\hline
\end{tabular}
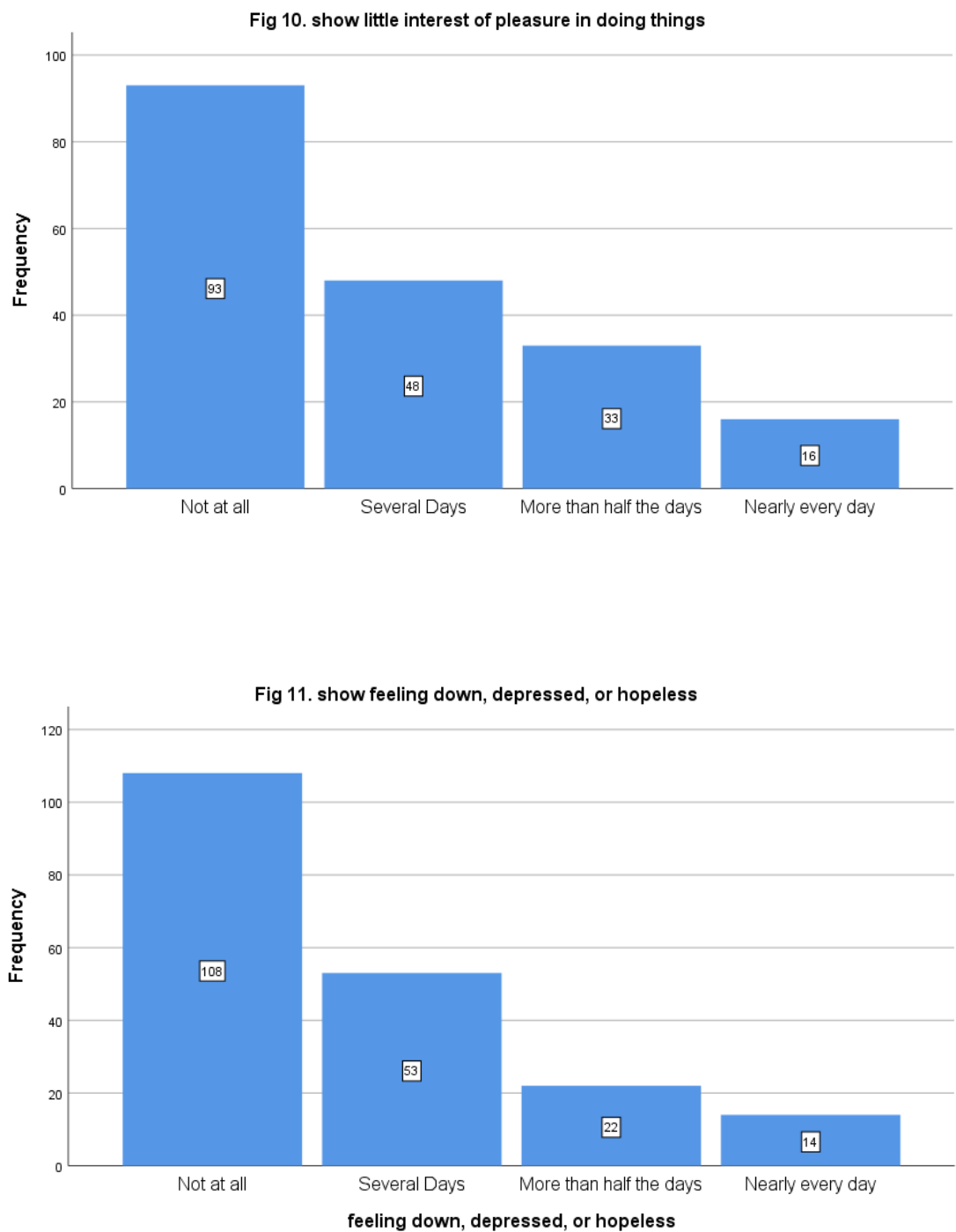

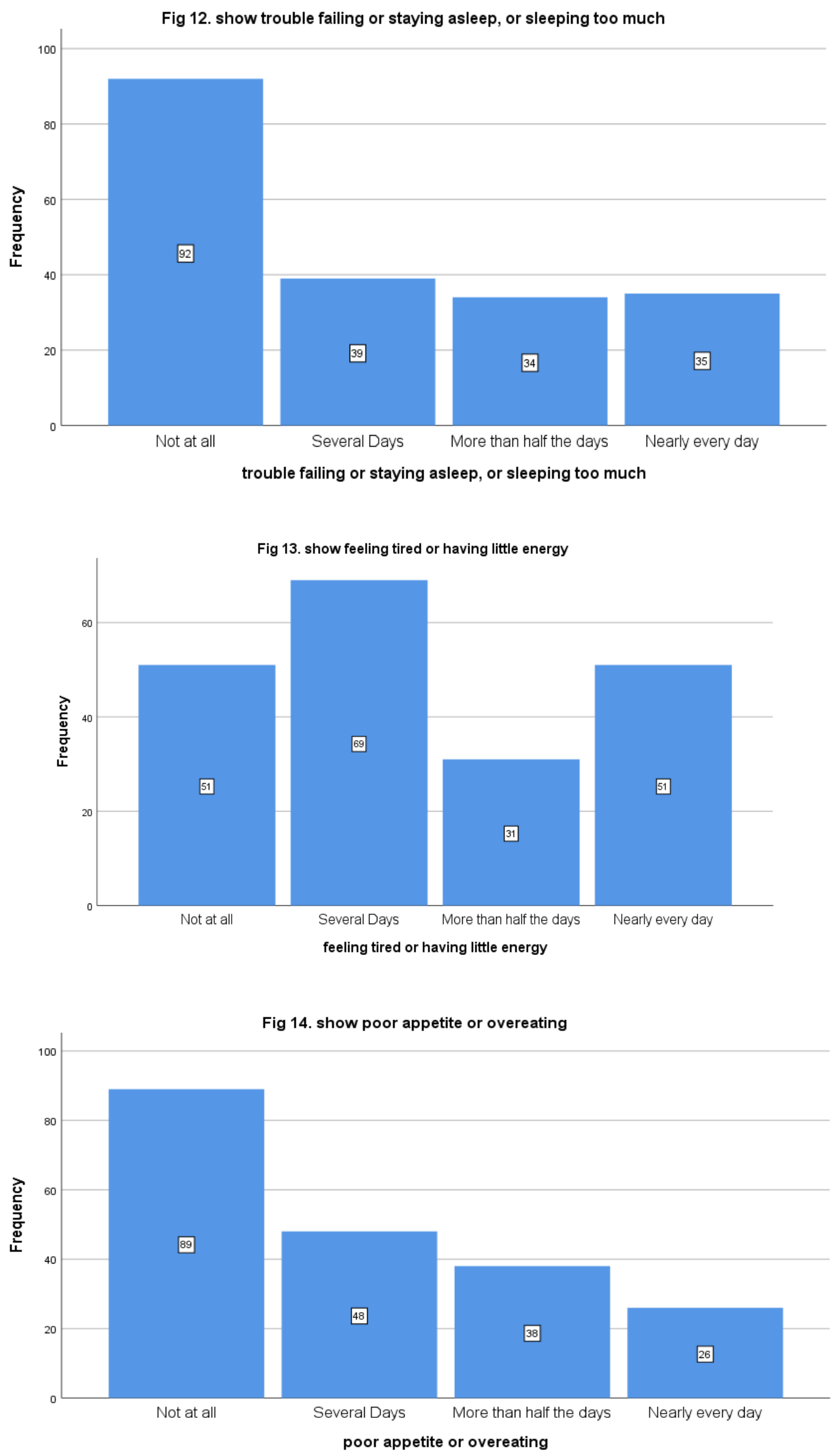
Fig 14. show feeling bad about yourself - or that you are a failure or have let yourself or your family down

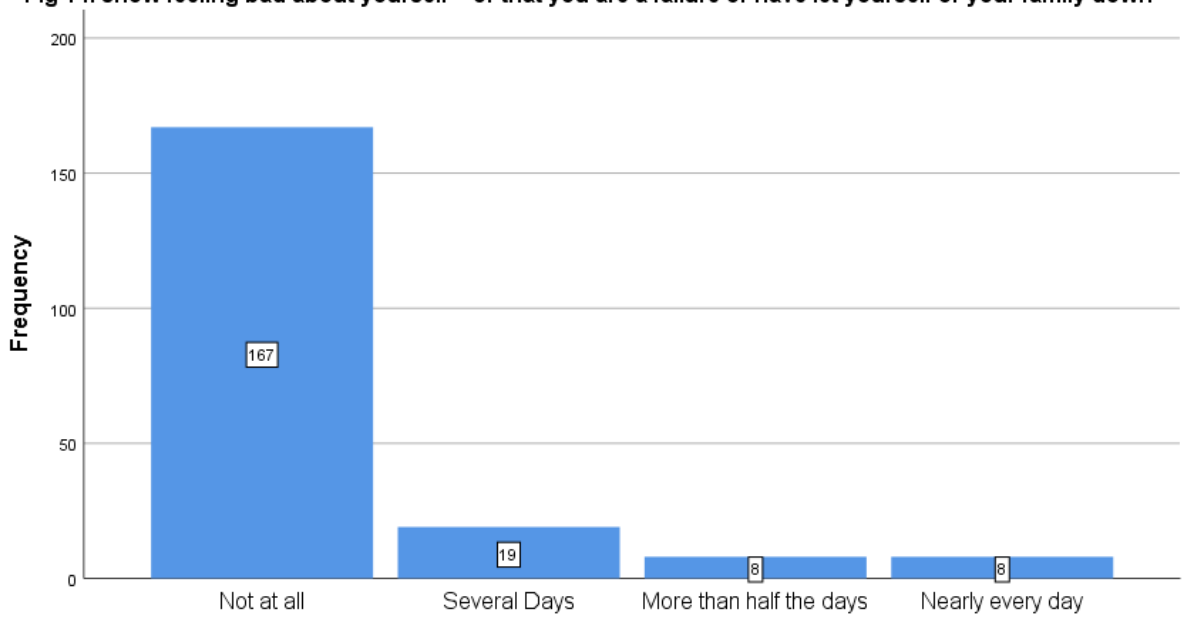

feeling bad about yourself - or that you are a failure or have let yourself or your family down

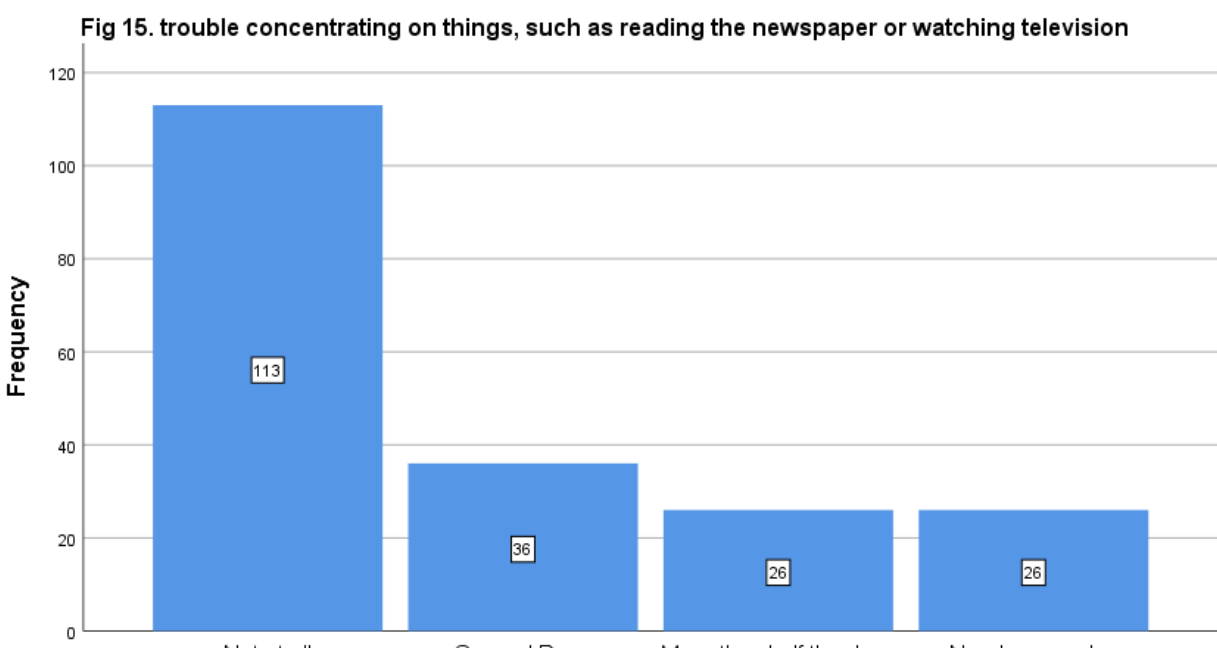

Not at all

Several Days

More than half the days

Nearly every day

trouble concentrating on things, such as reading the newspaper or watching television

Fig 16. show moving or speaking so slowly that other people could have noticed. Or the opposite - being so figety or restless that you have been moving around a lot more than usual

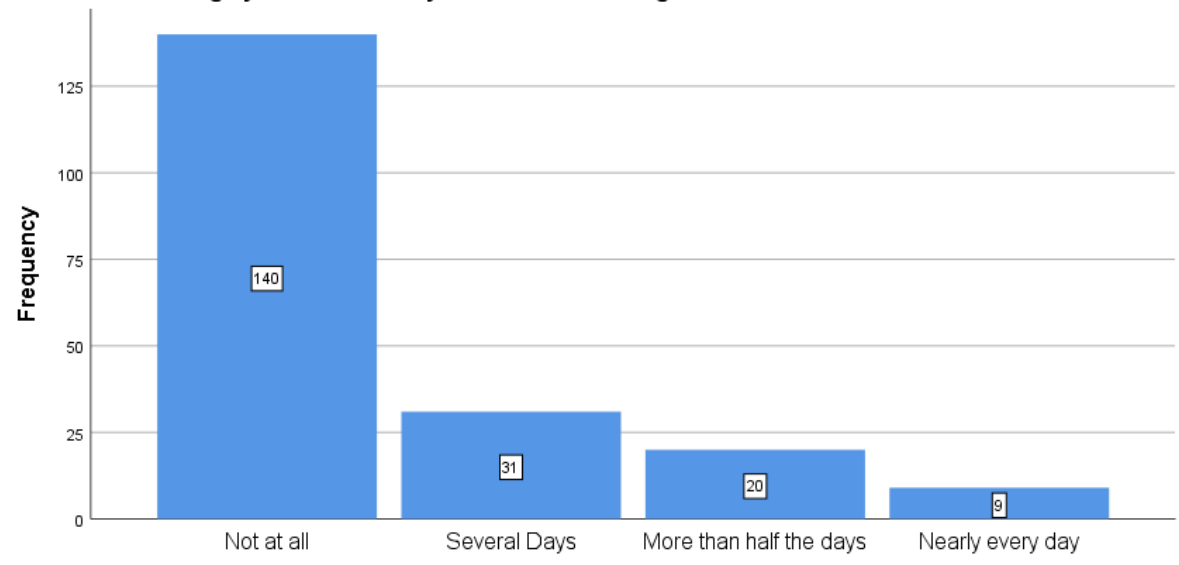

moving or speaking so slowly that other people could have noticed. Or the opposite - being so figety or restless that you have been moving around a lot more than usual 


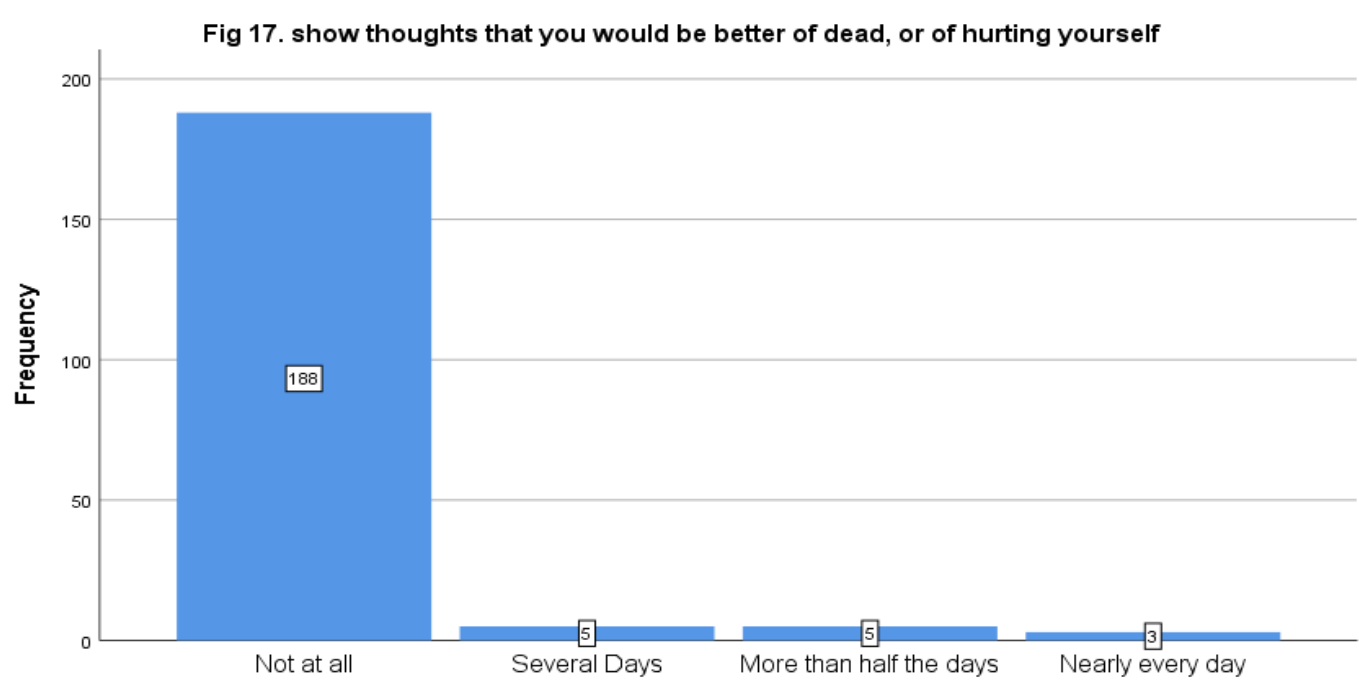

thoughts that you would be better of dead, or of hurting yourself

Fig 18. show If you checked off any problems, how difficult have these problems made it for you to do your work, take care of things at home, or get along with other people?

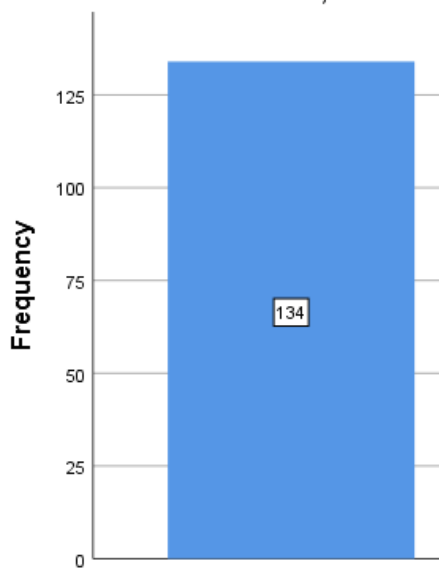

There is no difficulty

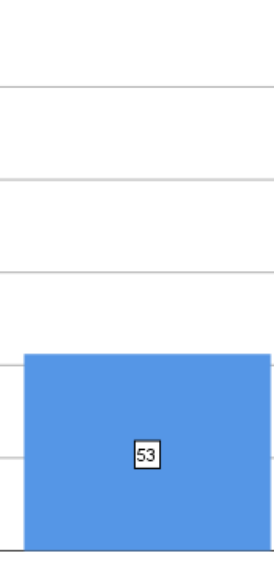

Pretty tough

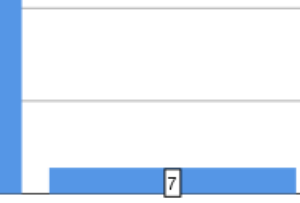

Very difficult

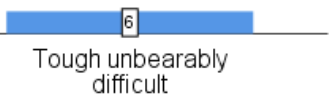

If you checked off any problems, how difficult have these problems made it for you to do your work, take care of things at home, or get along with other people?

Fig 10. show the degree of depression

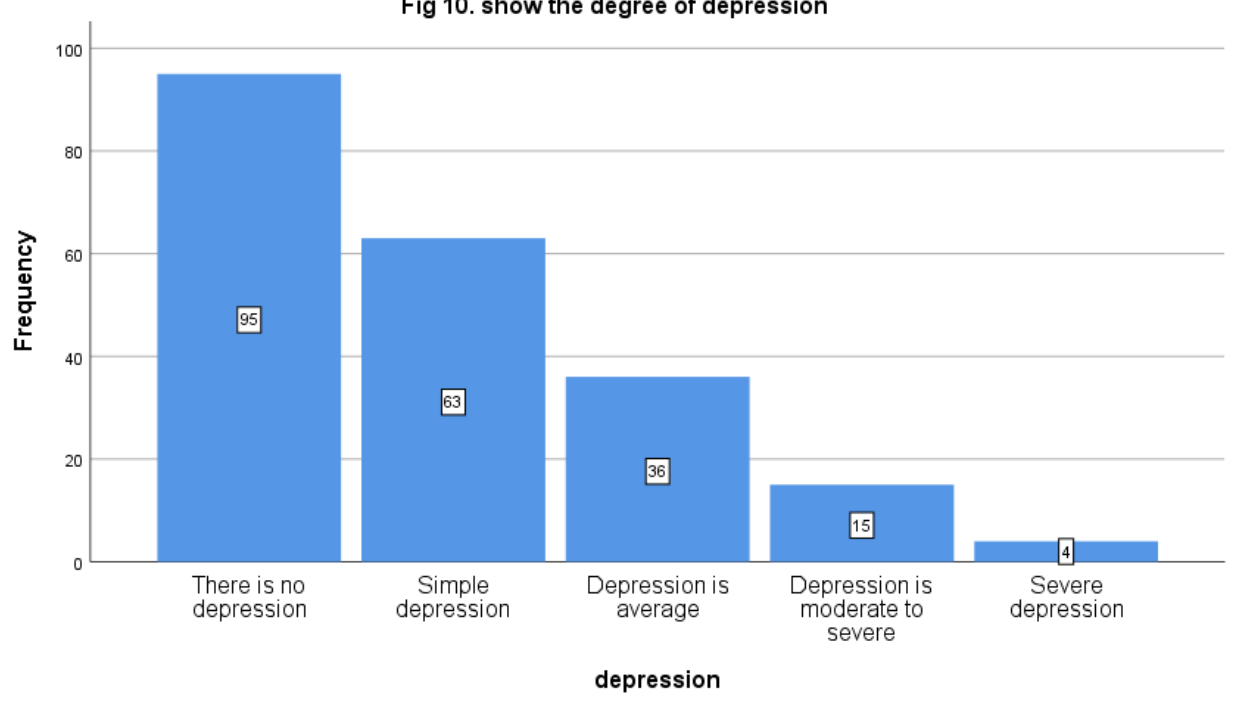




\section{Discussion}

In this study, depression was more prevalent among diabetic patients, where $55 \%$ were found to have depression. According to depression severity, mild depression was the most common among patients $(26.2 \%)$ followed by moderate depression $(16.9 \%)$ them moderate to severe $(7 \%)$, whereas severe depression was found among $1.9 \%$ of patients only. A study from Al Qassim, $\mathrm{KSA}^{[29]}$, showed that $34.8 \%$ of T2diabetic patients had depression; the prevalence was lower than that in our findings. Another study from Eastern Province, Saudi Arabia showed a prevalence close to our results, where depression was prevalent among $49.6 \%$ of diabetic patients ${ }^{[28]}$.

A study from Ethiopia using PHQ-9 questionnaire reported that the prevalence of depression among T2DM outpatients was $13 \%, 28.4 \%$ had mild depression, $12.1 \%$ had moderate depression, $2.7 \%$ and $1.5 \%$ had moderately severe and severe depression respectively ${ }^{[34]}$. A study from Chandigarh, India found that $41 \%$ of type 2 diabetic patients had depression, 23\% severe depression and $18 \%$ had moderate depression ${ }^{[35]}$. A study from Jordan by Ahmad et $\mathrm{al}^{[36]}$ used PHQ-9 questionnaire to investigate depression in diabetic foot patients and it was found that the depression prevalence was $39.6 \%$. One study from India used PHQ-9, but was performed on patients with both T1DM and T2 DM, the depression prevalence was found to be $35 \%$ among diabetic patients ${ }^{[37]}$. It was reported in Indian study by Mishra et $\mathrm{al}^{[33]}$ that the depression prevalence among diabetic patients was $43.33 \%$ and among those depressed patients, there were $30.76 \%$ suffering mild depression, $40.76 \%$ suffering moderate depression and $28.46 \%$ suffering severe depression, these percents were higher than percents found in our results, this can be attributed to the very poor controlled diabetes. A study from India showed lower prevalence of depression among patients with T2DM, only $9 \%$ of patients had depression, this may attributed to the difference in the life style in each region in india $^{[38]}$. Another Indian study ${ }^{[39]}$ showed higher depression prevalence of $28.3 \%$. The current study showed that, depression was significantly associated with older age $(\mathrm{P}$-value $=0.005)$, where depression was prevalent in $(57.4 \%)$ of those with age older than 45 and it was less prevalent in those with younger age; $48.6 \%$ of those with $25-45$ years old had depression and $33.3 \%$ only had depression of those less than 25 years old. Also, depression was associated with female gender ( $\mathrm{P}$ value $=0.005$ ), where higher percent of females $(68.2 \%)$ had depression and lower percent had no depression (31.8\%). Both marital and employment status had no impact on the prevalence of depression among our patients (P-value $>0.05$ ), whereas educational level had a significant impact on the prevalence of depression $(\mathrm{P}$-value $=0.006$ ). Patients with elementary education were more prone to suffer depression than graduated ones (70.2vs 14.3 having depression respectively). Also, the monthly income was another factor that significantly associated with depression (Pvalue $=0.000$ ), depression was more prevalent among patients with lower income, increasing monthly income made the depression less prevalent. Regarding clinical characters of our patients, it was found that type of treatment had no influence on the depression prevalence among diabetic patients $(\mathrm{P}$-value $=0.2)$, while both time of diagnosis and presence of chronic disease significantly associated with the prevalence of depression (P-value $=0.023,0.027$ respectively). Depression was more common among patients diagnosed with diabetes since5-10 years (60.3\%) and it was more prevalent among patients suffering chronic diseases including; hypothyroidism $(87.5 \%)$, followed by high blood lipid $(66.7 \%)$ and rheumatism $(66.3 \%)$. A study from Bangladesh demonstrated that the significant predictors of depressive symptoms among T2 diabetic outpatients using PHQ-9 were age, income, gender and cardiovascular disease [40]. A study from India showed that duration of diabetes and family income had no impact on the level of depression (P-value=0.1, 0.06 respectively), whereas health problems and 
exercise were significantly influenced depression level (P-value $=0.002,0.003$ respectively), where depression was more common in those with health problems and who didn't perform exercise ${ }^{[38]}$. In accordance with our findings, the prevalence of depression was associated with female gender (Pvalue $=0.01$ ) as reported in Jordanian study, however the opposite was found regarding age, where depression was associated with age younger than 50 years old [36]. One study ${ }^{[37]}$ demonstrated that higher prevalence of depression was found among type 2diabetic patients who had hypertension ( $\mathrm{P}$-value=0.022), while in our study the depression was higher among patients with hypothyroidism, also in contrast to our results the authors reported that age and duration of diabetes had no significant association with depression, however in accordance with our findings, unemployed individuals were the most to suffer depression $(\mathrm{P} \text {-value }=0.023)^{[37]}$.

\section{Conclusion}

Depression prevalence among T2DM patients is higher in the current study than other previous Saudi studies and the factors influencing the depression prevalence were varied among previous studies and our study. This may explained by the difference in the tool used to assess the depression in some studies as well as the population characteristics which differ among different area. We can conclude that depression and $\mathrm{T} 2 \mathrm{DM}$ are associated to each other and different factors can enhance the prevalence of depression.

\section{Acknowledgment}

Special Thanks for Allah and my Family for their Support and everything they made for me.

Special thanks to Dr Jamal Al Hamad for helping shape the project proposal and for all his support during the Program.

Finally I can't forget my entire colleague on Family Medicine Program for their Support.

\section{References}

1. Mirghani HO, Mohammed OS, Saadallah AM. Prevalence of depression among Sudanese patients with type-2 diabetes mellitus. Prevalence. 2014; 1;9(3).

2. International Diabetes Federation. Diabetes Atlas, 4th edition. 2009. Available from: http://www.worlddiabetesfoundation.org/c o mposite-35.htm. [cited 2015 Jan].

3. Al-Rubeaan K, Al-Manaa HA, Khoja TA, Ahmad NA, Al-Sharqawi AH, Siddiqui K, et al. Epidemiology of abnormal glucose metabolism in a country facing its epidemic: SAUDI-DM study. J Diabetes. 2014 Sep 30.

4. WHO. Global report on diabetes executive summary [Internet]. Available from: http://apps.who.int/iris/bitstream/10665/ 204871/1/9789241565257_eng.pdf.

5. International diabetes federation. IDF Diabetes Atlas. Brussels, Belgium: International diabetes federation, 2011.

6. Thour A, Das S, Sehrawat T, Gupta Y. Depression among patients with diabetes mellitus in North India evaluated using patient health questionnaire-9. Indian journal of endocrinology and metabolism. 2015 Mar;19(2):252.

7. Sweileh WM, Abu-Hadeed HM, Al-Jabi SW, Sa'ed HZ. Prevalence of depression among people with type 2 diabetes mellitus: a cross sectional study in Palestine. BMC public health. 2014 Dec;14(1):163.

8. World Health Organization. Depression Fact sheet N8 369. 2012. Available at: http://www.who.int/mediacentre/factsheets /fs369/en/. Accessed May 12, 2016.

9. Al-Khathami AD, Ogbeide DO. Prevalence of mental illness among Saudi adult primary-care patients in Central Saudi Arabia. Saudi Med J 2002; 23(6): $721 \mathrm{e} 724$. 
10. Anderson RJ, Freedland KE, Clous RE, Lustman PJ. The prevalence of comorbid depression in adults with diabetes: a metaanalysis. Diabetes Care 2001; 24: 10691078.

11. Ali S, Stone MA, Peters JL, et al. The prevalence of co-morbid depression in adults with type 2 diabetes: a systematic review and meta-analysis. Diabet Med 2006; 23: 1165-1173.

12. Dunbar JA, Reddy P, Davies-Lameloise N, et al. Depression: an important comorbidity with metabolic syndrome in a general population. Diabetes Care 2008; 31: 2368-2373.

13. Egede LE, Zheng D, Simpson K. Comorbid depression is associated with increased health care use and expenditures in individuals with diabetes. Diabetes Care 2002; 25: 464-470.

14. Medved V. The comorbidity of diabetes mellitus. Psychiatria Danubina 2009; 21(4): 585e588.

15. Koenig HG, Al Zaben F, Sehlo MG, Khalifa DA, Al Ahwal MS, Qureshi NA, et al. Mental health care in Saudi Arabia: past, present and future. Open J Psychiatry [Internet] 2014; 4(2): 113e130.

16. Rotella F, Mannucci E. Depression as a risk factor for diabetes: a meta-analysis of longitudinal studies. J Clin Psychiatry. 2013;74: 31-37.

17. Campayo A1, de Jonge P, Roy JF. Depressive disorder and incident diabetes mellitus: the effect of characteristics of depression. Am J Psychiatry. 2010 May; 167(5):580-8.

18. Williams MM, Clouse RE, Lustman PJ. Treating depression to prevent diabetes and its complications: Understanding depression as a medical risk factor. Clin Diabetes 2006;24:79-86.

19. Chen PC, Chan YT, Chen HF, et al. Population-based cohort analyses of the bidirectional relationship between type 2 diabetes and depression. Diabetes Care. 2013;36:376-382.

20. Golden SH, Lazo M, Carnethon M, et al. Examining a bidirectional association between depressive symptoms and diabetes. JAMA. 2008;299:2751-2759.

21. Pan A, Lucas M, Sun Q, et al. Bidirectional association between depression and type 2 diabetes mellitus in women. Arch Intern Med. 2010;170:18841891.

22. AlHadi AN, AlAteeq DA, Al-Sharif E, Bawazeer HM, Alanazi H, AlShomrani AT, Shuqdar RM, AlOwaybil R. An arabic translation, reliability, and validation of Patient Health Questionnaire in a Saudi sample. Annals of general psychiatry. 2017 Dec;16(1):32.

23. Ali N, Jyotsna VP, Kumar N, Mani K. Prevalence of depression among type 2 diabetes compared to healthy non diabetic controls. J Assoc Physicians India. 2013 Sep;60:31-3.

24. Lunghi C, Moisan J, Grégoire JP, Guénette L. Incidence of depression and associated factors in patients with type 2 diabetes in Quebec, Canada: a population based cohort study. Medicine. 2016 May;95(21).

25. Lustman PJ, Griffith LS, Clouse RE. Depression in adults with diabetes: Results of 5-yr follow-up study. Diabetes Care 1988;11:605-612.

26. Egede LE, Zheng D. Independent factors associated with major depressive disorder in a national sample of individuals with diabetes. Diabetes Care 2003;26:104-111.

27. Bensbaa S, Agerd L, Boujraf S, Araab C, Aalouane R, Rammouz I, et al. Clinical assessment of depression and type 2 diabetes in Morocco: Economical and social components. J Neurosci Rural Pract. 2014; 5(3):250-3.

28. El Mahalli AA. Prevalence and predictors of depression among type 2 diabetes mellitus outpatients in Eastern Province, 
Saudi Arabia. International journal of health sciences. 2015 Apr;9(2):119.

29. Al-Mohaimeed AA. Prevalence and factors associated with anxiety and depression among type 2 diabetes in Qassim: A descriptive cross-sectional study. Journal of Taibah University Medical Sciences. 2017 Oct 1;12(5):430-6.

30. De Groot M, Anderson R, Freedland KE, et al. Association of depression and diabetes complications: a meta-analysis. Psychosom Med. 2001;63:619-630.

31. Lin EH, Rutter CM, Katon W, et al. Depression and advanced complications of diabetes: a prospective cohort study. Diabetes Care. 2010;33:264-269.

32. Lustman PJ, Anderson RJ, Freedland KE, et al. Depression and poor glycemic control: a meta-analytic review of the literature. Diabetes Care. 2000;23:934942.

33. Mishra AK, Kumar S, Ahmad A, Kumar G, Singh KK, Saha KK, Kumar A, Kumar R. Prevalence of Depression in Diabetes Mellitus and Its Determinants. International Journal of Scientific Study. 2017 Jul 1;5(4):151-4.

34. DejenieHabtewold T, Radie YT, Sharew NT. Prevalence of depression among type 2 diabetic outpatients in black lion general specialized hospital, Addis Ababa, Ethiopia. Depression research and treatment. 2015;2015.

35. A. Raval, E. Dhanaraj, A. Bhansali, S. Grover, and P. Tiwari, "Prevalence \& determinants of depression in type 2 diabetes patients in a tertiary care centre," Indian Journal of MedicalResearch;2010: 132( 8): 195-200.

36. Ahmad A, Abujbara M, Jaddou H, Younes NA, Ajlouni K. Anxiety and Depression
Among Adult Patients With Diabetic Foot: Prevalence and Associated Factors. Journal of clinical medicine research. 2018 May;10(5):411.

37. Paulraj S, Rajasekaran S \& Mathew AC. PREVALENCE OF DEPRESSION AND ITS ASSOCIATED FACTORS IN ADULTS WITH DIABETES MELLITUS: A CROSS-SECTIONAL STUDY IN A TERTIARY CARE CENTRE. Advanced Medical Sciences: An International Journal (AMS); 2017:4(1/2/3).

38. Dahal P,Balamurugan G \&Basavaraja AU. Prevalence and Predictors of Depression among Diabetes Mellitus in Adult Population. J Psychiatry 2017, 20:6.

39. Nawaz AS, Mane AB, Ramakrishna MR. Diabetes and depression: Impact of depression on self-care among patients with type 2 diabetes. International Journal of Medical Science and Public Health. 2015 Jul 1;4(7):901-5.

40. Roy T, Lloyd CE, Parvin M, Mohiuddin KG, Rahman M. Prevalence of co-morbid depression in out-patients with type 2 diabetes mellitus in Bangladesh. BMC psychiatry. 2012 Dec;12(1):123.

\section{List of Abbreviation}

\begin{tabular}{|c|c|}
\hline Abbreviation & Definition \\
\hline$(\mathrm{DM})$ & Diabetes mellitus \\
\hline (T2DM) & Type 2 diabetes mellitus \\
\hline (PHQ) & patient health questionnaire \\
\hline SFH & Saudi Commission for Health \\
\hline SCFHS & $\begin{array}{l}\text { Saudi Commission for Health } \\
\text { Specialty }\end{array}$ \\
\hline
\end{tabular}

